Short Communication

\title{
THE RANGE OF POLLEX-INDEX APPROXIMATIONS IN OPPOSITION
}

\author{
Arunachalam Kumar \\ Professor of Anatomy, K. S. Hegde M edical Academy, Nitte University \\ M angalore - 575018 \\ Correspondence : \\ Arunachalam Kumar \\ Phone : 0824-2204490 E-mail : dean.kshema@ nitte.edu.in
}

\begin{abstract}
:
Pinch gripping is an evolved hominid attribute: the modification of the first carpo-metacarpal articulation into a sellar type enables an opposable thumb; The pinch, preferentially performed by approximation of the pollex-index fingertip pulps shows variation in range of purchase between these digits depending on the power of grip. This brief report analyzes the area of surface contact between the pollex and indextip in both, light and tight pinch-grip.
\end{abstract}

The possible role of index anatomy in enhancing the level of efficiency of pinch-grip in opposition is discussed.

Keywords : opposition, index, pollex, pinch grip, purchase area, pulp

\section{Introduction:}

The evolution of the highly complex, yet perfectly coordinated articulation at the first carpometacarpal joint, opposition, probably is of as much significance in the differentiation of the higher primates into hominids, as are the modifications in the pelvic and post-pelvic skeletal elements.

The performance of the uniquely human digital movement of opposition, is facilitated by the conversion of a once condylar joint into a saddle variety in the pollex (thumb). Whilst much attention has been given to this osseous change at the proximal pollex end, little, if at all any, has been concentrated on what has been recorded on the alteration, that opposition has induced in the 'favoured for in opposition' digit, the index. This paper records the fairly vital role the index performs in opposition kinetics.

\section{Materials \& Methods :}

110 individuals of normal health, belonging to all ages and both sexes were asked to perform opposition movement. Using inked stamping pad, stained index and pollex pulps of right and left hand of each were made to grip standard sized thick paper. Two impressions, one with light and another with hard (tight) grip were taken. Two records from each hand per individual were made. The transverse (breadth) of each imprint was measured in mms. The resultant data was tabulated and analyzed.

\section{Observations:}

From data collected it was observed, consistently, that for every $1 \mathrm{~mm}$ increment in the transverse dimension of the pollex pulp in tight grip, the index pulp exercised a roll of 3 mms.

With minor variations, the ratio of $1: 3$ was maintained for tight grip, in all age groups and in both sexes. In this paper we are presenting a part of the exhaustive data compiled, only restricting to the measurably-clear records made in 152 impressions of right hand digits in 76 individuals of both sexes, of adult age groups.

\section{Discussion :}

Physical anthropologists have long been fascinated by thumb biomechanics ${ }^{1}$. The exclusively human opposition and its mastery, is landmark development in the evolutionary tree. It is evident that for the best performance of opposition, the preferred adjunct digit is the index ${ }^{2,3,5}$. The fact that the index takes a crucial role in tight gripping proves that in some anatomical manner ${ }^{4}$, this digit has probably adapted itself to the requirements of it's partner for opposition, Kumar ${ }^{6,7}$ first reported that the index in it's rest or anatomical position, lies in an axis that is rotated radiad and that this in-situ rotated status confers on it, the capacity to augment opposition power. The pre- 
opposed index is so positioned, that on reception of the pollex pulp in tight grip, the purchase area (transverse of approximated surface) exhibits an inbuilt augmentative potential. (Figure \& Table)

\begin{tabular}{|l|l|l|}
\hline AGE & M ALE & FEM ALE \\
\hline $20-30$ & 3.12 & 3.44 \\
\hline $30-40$ & 2.9 & 3.18 \\
\hline $40-50$ & 3 & 4 \\
\hline $50-60$ & 3.33 & 2.75 \\
\hline $60-80$ & 3.4 & 3.22 \\
\hline $80+$ & 4.8 & 2.77 \\
\hline \multicolumn{3}{|l|}{$\begin{array}{l}\text { Movement of the Index (in mm) } \\
\text { for every } 1 \text { mm of the Thumb }\end{array}$} \\
\hline
\end{tabular}

\section{References :}

1. Arunachalam Kumar, The Index digit rotation and opposition evolution, Human Races M onthly, Vol. 1, No.9, 2002

2. Rao A \& Arunachalam Kumar, Mathematical Analysis of FlexionExtension of Index Digit, Proceedings of the N.C.B.E., Ed. UC Niranjan, VIII 21, 1998

3. Manou J \& Arunachalam Kumar, The Rotational Kinetics of IndexPollex in Opposition Exercise, $48^{\text {th }}$ A.S.I. National Conference, M anipal, 2001

4. Rao A \& Arunachalam Kumar A, Opposition Biomechanics: A Community Study, National Conference on Biomedical Engineering, Manipal, 1998
As an offshoot, could it be of value, were hand surgeons reimplanting amputated index digits, not in the accepted anteroposterior orientation but in the radially rotated positus?

\section{SURFACE AREA EXTENSIONSIN OPPOSITION \\ THUMB INDEX}

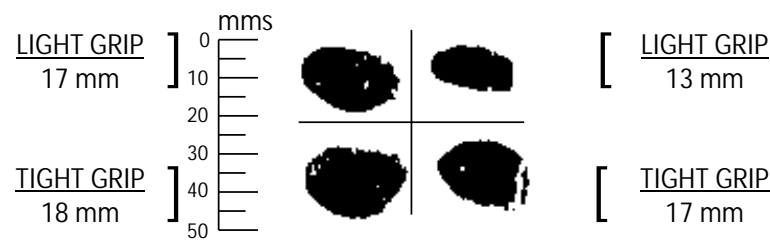

5. Rao A \& Arunachalam Kumar, Mathematical Analysis of FlexionExtension of Index Digit, Proceedings of the N.C.B.E., Ed. U C Niranjan, VIII 21, 1998

6. Savita $S \&$ Arunachalam Kumar, The Thumb-Index Complex Biomechanics in Opposition, XIV Conference of Indian Society of Hand Surgeons, M anipal, 1990

7. Arunachalam Kumar, Complementary Rotation of Index in ThumbIndex Opposition Proceedings of the International Conference of Biomechanics \& Clinical Kinesiology of Hand \& Foot, Eds. Patil K M \& Srinivasan H, IIT M adras, 1985 